\title{
Elimination of current sheets at resonances in three-dimensional toroidal ideal-mhd equilibria
}

\author{
M.I.Mikhailov ${ }^{1}$, J.Nührenberg ${ }^{2}$, and R.Zille ${ }^{3}$ \\ 1 National Research Centre "Kurchatov Institute", Moscow, Russia \\ 2 Max-Planck-Institut für Plasmaphysik, Wendelsteinstr. 1, 17491 Greifswald, \\ Germany \\ ${ }^{3}$ Max-Planck-Institut für Plasmaphysik, Boltzmannstr. 2, 85748 Garching, \\ Germany \\ E-mail: Juergen.Nuehrenberg@ipp.mpg.de
}

\begin{abstract}
On the basis of the ideal-mhd equilibrium code VMEC [Hirshman S et. al, 1986 Comp. Phys. Comm. 43 143] supplemented by transformation of its results into magnetic coordinates it is shown how current sheets, radially widened by the finite grid size, at rational values of rotational transform in 3d toroidal equilibria can be removed by variation of the equilibrium geometry.
\end{abstract}




\section{Introduction}

Recently it has been stated that the $3 \mathrm{~d}$ equilibrium code VMEC [1] cannot compute current sheets at rational values of rotational transform in $3 \mathrm{~d}$ toroidal mhd equilibria [2]. On the other hand, the existence of magnetic islands and current sheets in threedimensional slab-geometry equilibria [3] and screwpinch geometry $[4,5]$ has been proven. More recently [6], VMEC has been verified for force-free large-aspectratio circular cross-section equlibria against linear ideal-mhd equilibrium theory in the limit of nested surfaces. Here, it will be shown that an evaluation of the equilibrium results of toroidal equilibria with general 3d geometry obtained with VMEC can find the signature of current sheets, computationally radially widened by the finite radial grid size in calculating the equilibrium.

Boozer's coordinates [7] $s, \theta, \phi$ (flux label, poloidal and toroidal coordinate) are used for this purpose. Their construction from a VMEC equilibrium is described in [8]. In these coordinates, the covariant component of $\vec{B}, B_{s}$, has been named $\tilde{\beta}$ because the inhomogeneous part of its equation when formulated as a differential equation along fieldlines

$$
\sqrt{g} \vec{B} \cdot \nabla \tilde{\beta}=p^{\prime}\left(\sqrt{g}-V^{\prime}\right)
$$

is proportional to the derivative of the pressure with respect to the flux coordinate. Here, $g$ is the Jacobian, $V(s)$ the volume enclosed by the flux surface with label $s$.

The radial dependency of the homogeneous part of $\tilde{\beta}$ on rational magnetic surfaces, $\iota_{\text {period }}=n / m$, is proportional to $\delta\left(s-s_{r e s}\right)$ and is related to the force-free current density sheet necessary for rational magnetic surfaces to exist [9]. In order to describe $\tilde{\beta}$ completely, an equivalent expression, calculating it from the geometry directly, is used

$$
\tilde{\beta}=-\left(F_{T}^{\prime} g_{\phi s}+F_{P}^{\prime} g_{\theta s}\right) / \sqrt{g}
$$

where $g_{\phi s}$ and $g_{\theta s}$ are elements of the Jacobian and $F_{T}$ and $F_{P}$ toroidal and poloidal flux, respectively. This expression is computed for various radial grid sizes so that the contribution of the resonance can be extrapolated to infinite radial grid size. Then a suitable boundary coefficient of the equilibrium investigated can be selected to eliminate the resonant contribution.

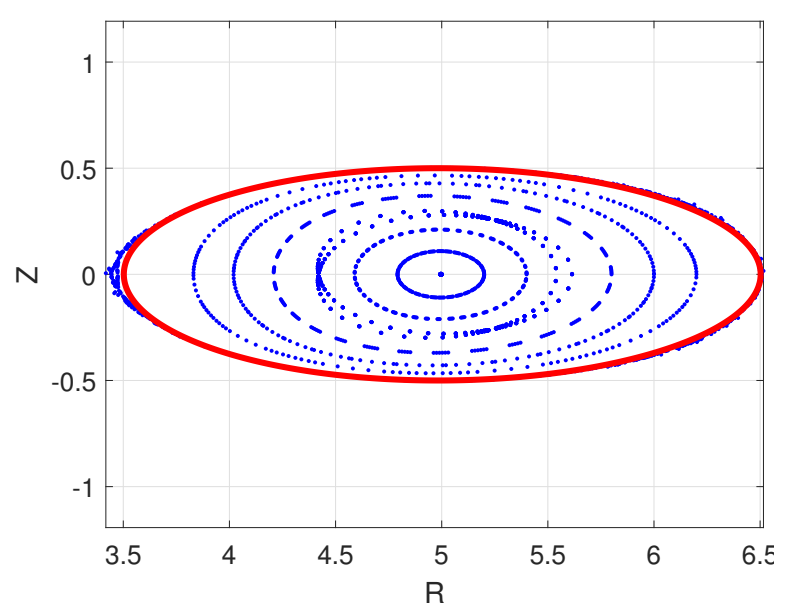

Figure 1. Poincaré plot of a three-period vacuum field. The non-vanishing boundary coefficients are: $R_{0,0}=5 ; R_{1,0}=$ $Z_{1,0}=1 ; R_{1,3}=-Z_{1,3}=0.5 ; R_{2,1}=-Z_{2,1}=0.0025$; solid line: VMEC boundary. In this configuration the rotational transform varies from $\approx 0.82$ at the center to $\approx 2.5$ at the boundary

\section{Case studies}

Three physically different types of configurations are investigated: a vacuum field, a force-free equilibrium, and a finite- $\beta$ equilibrium.

a) A vacuum field

If a vacuum field with an outer smooth magnetic surface and an inner island chain at a resonance is recomputed with VMEC as a zero- $\beta$ ideal-mhd (i.e. with infinite conductivity) no-net-current equilibrium with nested surfaces, a surface current density appears at the resonance. Here, this is demonstrated starting from a simple $l=2$ stellarator with 9 periods and rotational transform unity occurring in the confinement region $\left(R_{0,0}=5 ; R_{1,0}=Z_{1,0}=1, R_{1,1}=\right.$ $\left.-Z_{1,1}=0.5\right)$. This configuration is changed into a 3 period configuration by an $m=3$ perturbation, threeperiodic on the torus (and thus resonant at $\iota=1$ ) so that an $m=3$ island chain appears at $\iota=1$. Figure 1 shows a cross-section of the VMEC boundary and the vacuum field solution for this boundary as obtained from an outside surface current density [10].

Figure 2 shows the resonant component of $\tilde{\beta}$ as obtained from VMEC. Its properties are characterized with three different evaluations. While the integrals of $\tilde{\beta}_{31}$ between its zeroes closest to the resonance converge, the amplitude of $\tilde{\beta}_{31}$ strongly grows with the 


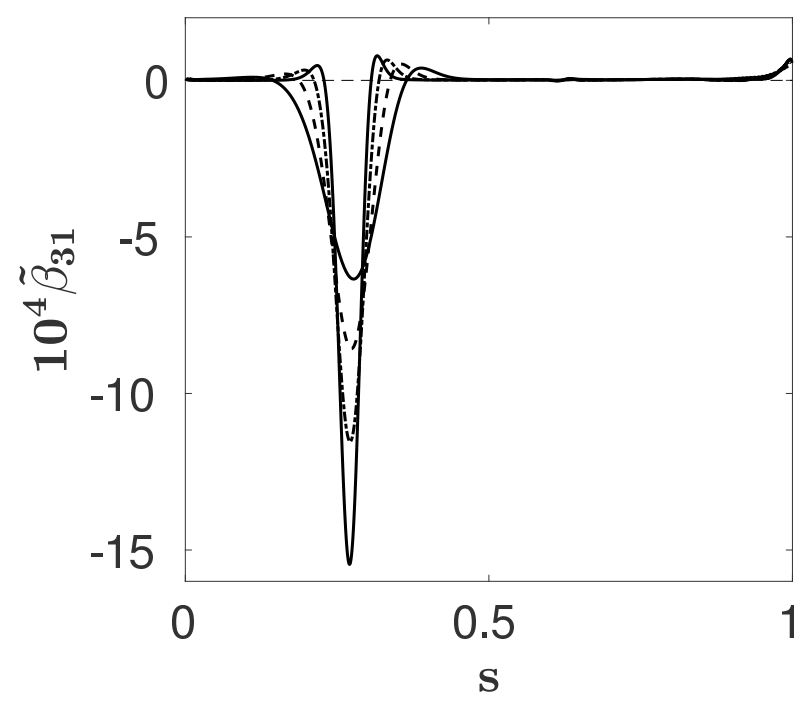

Figure 2. $\tilde{\beta}(m=3, n=1)$ as a function of $s$ for various gridsizes $(307,613,1223,2447)$ in VMEC. The singular behaviour of $\tilde{\beta}(m=3, n=1)$ becomes sharper with increasing radial grid size $n s$.

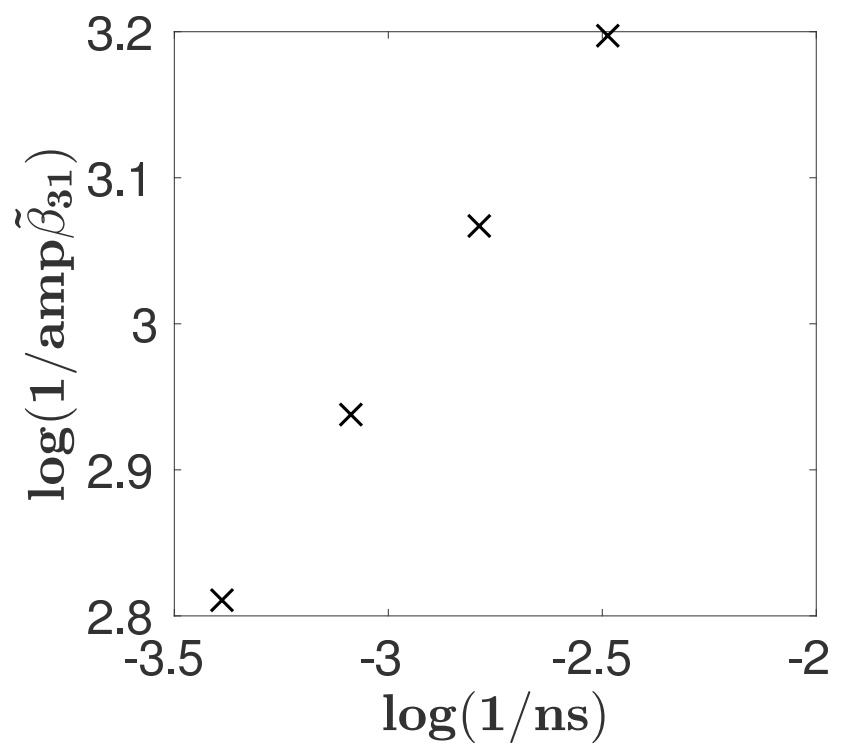

Figure 3. The inverse of the amplitude of the $m=3, n=1$ Fourier component of $\tilde{\beta}$ as a function of mesh widths in the vacuum field case.

mesh size and the width of $\tilde{\beta}_{31}$ at half of its amplitude strongly shrinks, see Figs. 3 and 4 .

These results indicate the interpretation that the singular behavior found is of $\delta$-function character.

From the point of view of selecting a viable configuration, perturbations that destroy the periodicity of a configuration must be avoided or precautions taken $[11]$.

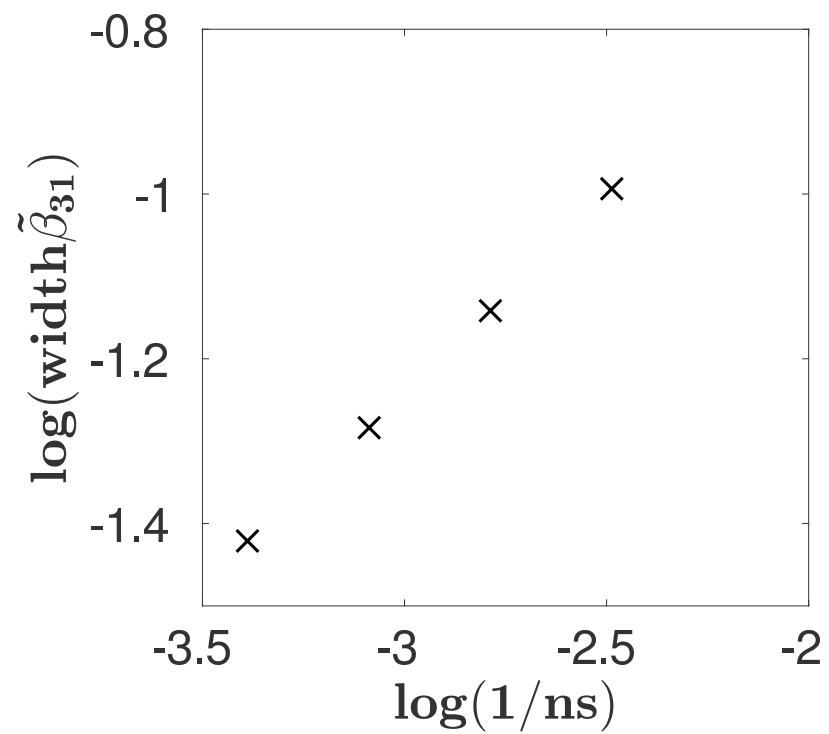

Figure 4. The width of the resonant component of $\tilde{\beta}$ at half of its amplitude as a function of mesh widths in the vacuum field case.

b) Force-free equilibrium

A two-period quasi-axisymmetric configuration [12], a tokamak-stellarator hybrid, is selected because low-order resonances (e.g. $\iota$ per period, $\iota_{p}=1 / 3$ ) occur which should be of particular importance. Its $\beta=0$ equilibrium is investigated with fixed rotational transform profile $(\iota=0.91-0.59 s)$ at its $\iota=$ $2 / 3$ resonance. Again, the signature of a $\delta$-function behaviour of $\tilde{\beta}_{31}$ smoothed by finite radial grid sizes is clearly seen in Fig. 5 .

A systematic search for a parameter of the equilibrium boundary [12] $\ddagger$ which is effective in reducing the amplitude of $\tilde{\beta}_{31}$ at the resonance is not laborious because the number of boundary coefficients is small. Since $m=2$ coefficients correspond to triangular deformations these coefficients should be tested first and yielded the boundary coefficient $Z(m=$ $2, n=0)$ whose values for eliminating the singularity in $\tilde{\beta}(m=3, n=1)$ (i.e. rendering it locally odd) only weakly depend on radial grid size, see Fig. 6, and turn out to converge quadratically with the grid-point distance to 0.1126 .

One expects that the shape of the magnetic surfaces should reflect a resonant current sheet and its elimination. This is seen in Fig. 7 where the two cases are compared with respect to $|\nabla s|^{2}$ : elimination of the current sheet eliminates the resonance signature in the $\mathrm{m}=3, \mathrm{n}=1$ Fourier component of $|\nabla s|^{2}$.

The change of the geometry of the configuration

$\ddagger$ In Table I the published version contains a misprint: $R(2,-1)$ should be 0.026 . 


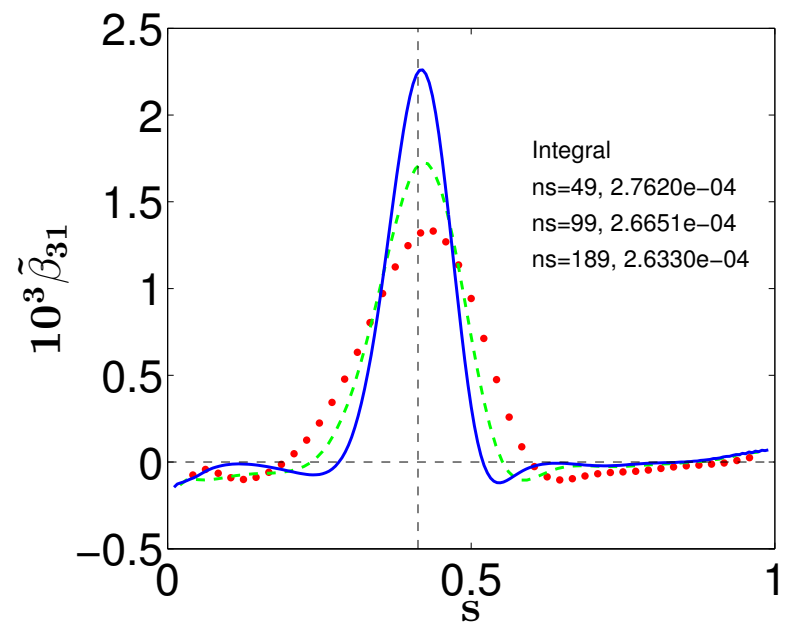

Figure 5. The $m=3, n=1$ Fourier component of $\tilde{\beta}$ as a function of the flux label for various grid sizes in the force-free case. The insets show the values of the integrals between the zero values adjacent to the resonance $2 / 3$ at $s=0.4123$. Here (and in Figs. 6 and 10) dotted, dashed and solid lines correspond to increasing mesh size.

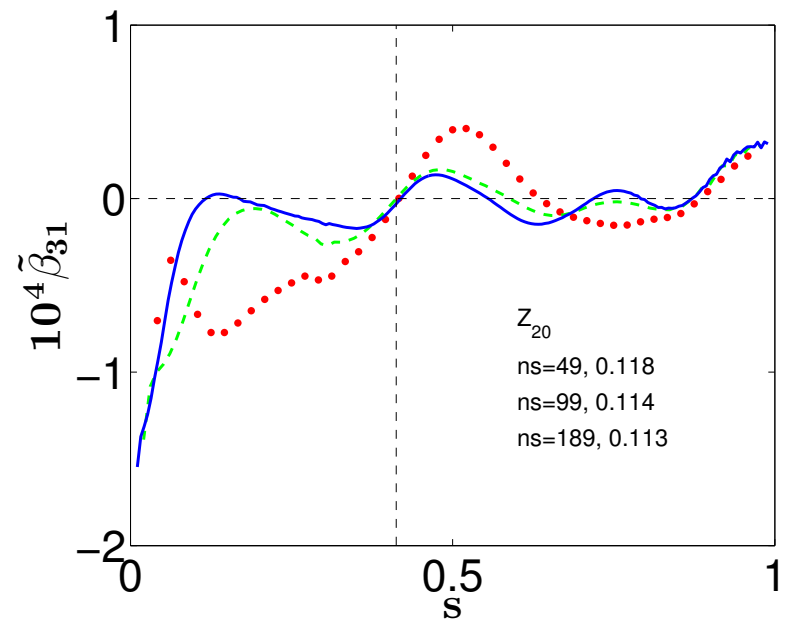

Figure 6. The same as in Fig. 5, now for various grid sizes and associated values of $Z_{m=2, n=0}$ (given in the inset) which render $\tilde{\beta}$ a locally odd function at the resonance. Please note the difference in vertical scale between Fig. 5 and here.

is shown in Fig. 8 where the flux surface crosssection is shown in that symmetry plane in which the smallest deviation from a circular cross occurs: a small reduction in triangularity and a small shift of the magnetic axis are seen.

c) Finite-pressure equilibrium

For 3D equilibria obtained under the assumption of nested flux surfaces at finite $\beta$, a stronger divergence than above dominates the behavior of the equilibrium at a resonance. The parallel current density exhibits a $1 /\left(s-s_{r e s}\right)$ singularity if the pressure gradient does

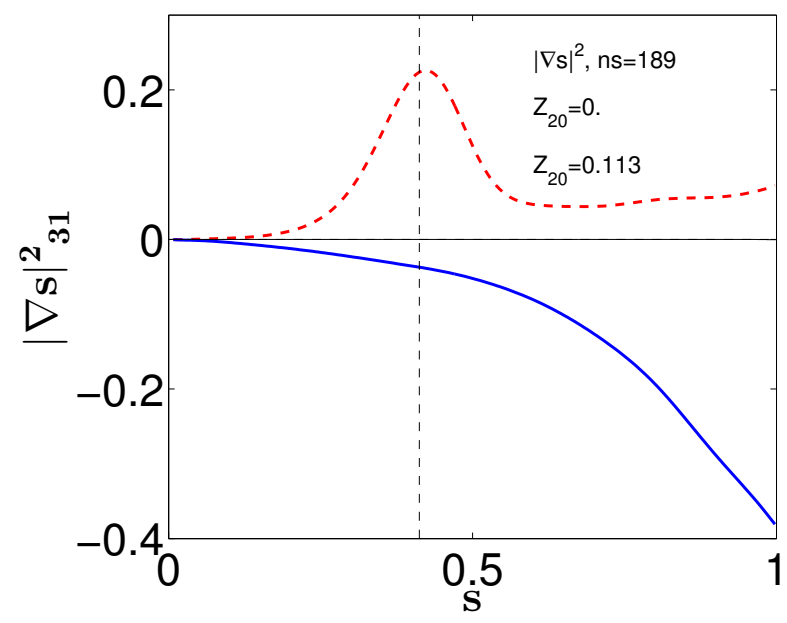

Figure 7. The $m=3, n=1$ Fourier component of $|\nabla s|^{2}$ as a function of the flux label without (dashed) and with (solid) elimimation of the resonance in the force-free case.

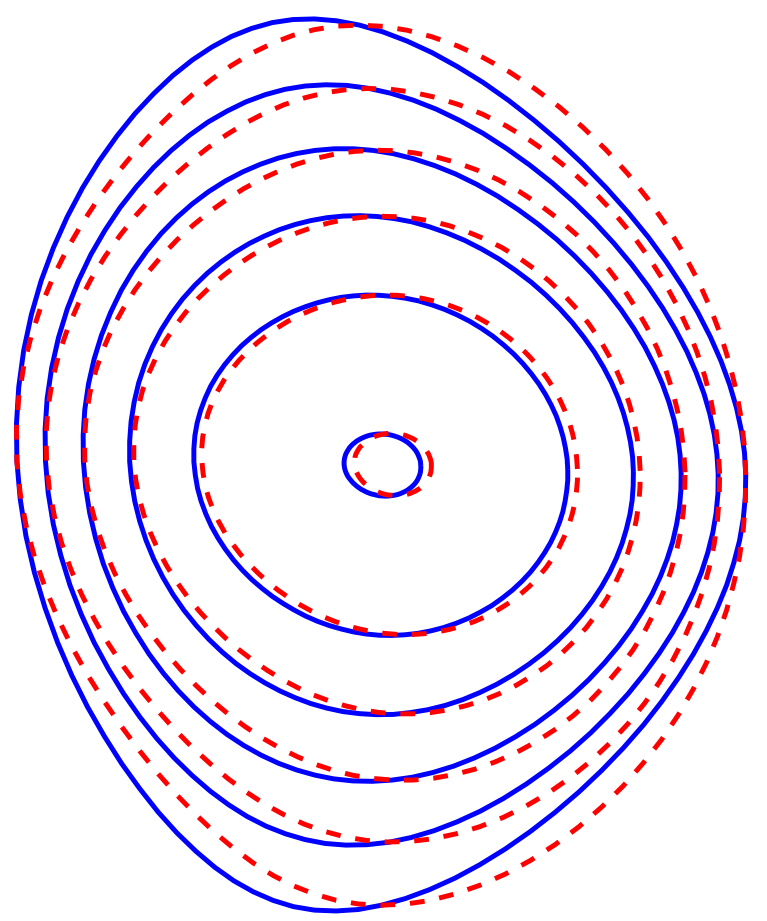

Figure 8. Flux surfaces without (solid) and with (dashed) the choice of $\mathrm{Z}(\mathrm{m}=2, \mathrm{n}=0)$ which eliminates the surface current density at the resonance 


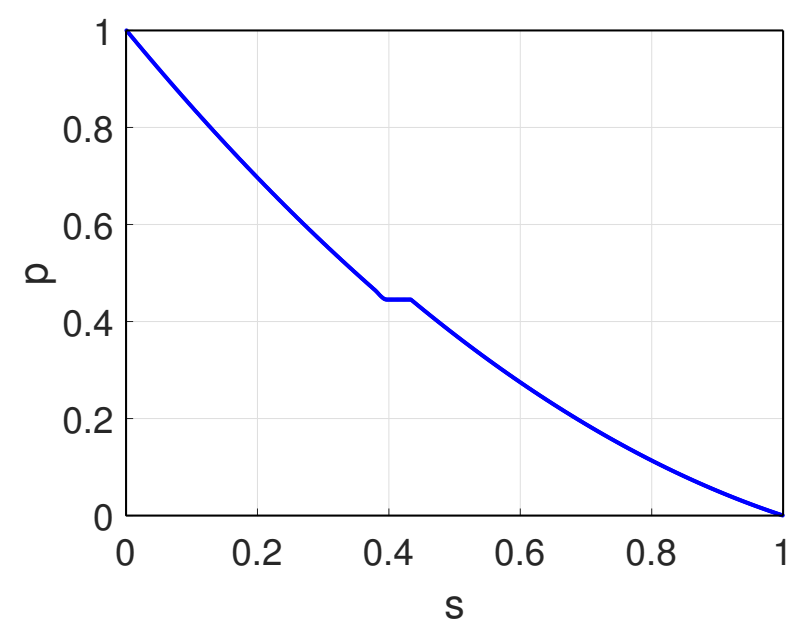

Figure 9. Normalized pressure profile for the finite- $\beta$ case.

not vanish [8].

The following procedure to deal with this situation is chosen. A pressure profile flattening as seen in Fig. 9 is introduced for the equilibrium calculation and eliminates the pressure-driven parallel-current-density divergence. The width of the flattening can be chosen such that, e.g. the Mercier stability criterion [13], is not violated near the resonance. Thus, a useful estimate is obtained on the importance of the resonance for the mhd stability of the configuration. Here, with the width of the flattening chosen, the Mercier stability criterion becomes approximately marginal at the boundaries of the flattening so that Mercier instability would occur for a significantly narrower pressure profile flattening.

The inhomogeneous solution for $\tilde{\beta}$ then exhibits a resonant Fourier component which vanishes in the immediate neighbourhood of the resonance and is approximately odd outside the small pressure-flattened region. Therefore, vanishing of the even part of the solution (the $\delta$-function widened by the finite grid size) is indicated by the local oddness of the resonant Fourier component.

Figure 10 shows results at $\langle\beta\rangle \approx 0.03$ for the same configuration as considered in case study $b$. The change of the boundary coefficient $Z_{20}$ needed to obtain the above property is similar to the one for $\beta=0$ and converges linearly to $Z_{20}=0.09$.

Since for case studies b) and c) a fixed profile of the rotational transform has been used, the toroidal current $J$ and the parallel current density are considered for completeness of the description. The parallel current density is given by $[8]$

$$
\begin{aligned}
\vec{j} \cdot \vec{B} / B^{2}= & \left(-J \tilde{\beta}_{\phi}+I \tilde{\beta}_{\theta}\right) /\left(F_{T}{ }^{\prime} I+F_{P}{ }^{\prime} J\right) \\
& +\left(J I^{\prime}-I J^{\prime}\right) /\left(F_{T}{ }^{\prime} I+F_{P}{ }^{\prime} J\right)
\end{aligned}
$$

so that it is conveniently discussed in terms of $J$ and $\tilde{\beta}$. Fig. 11 shows the toroidal currents in the most

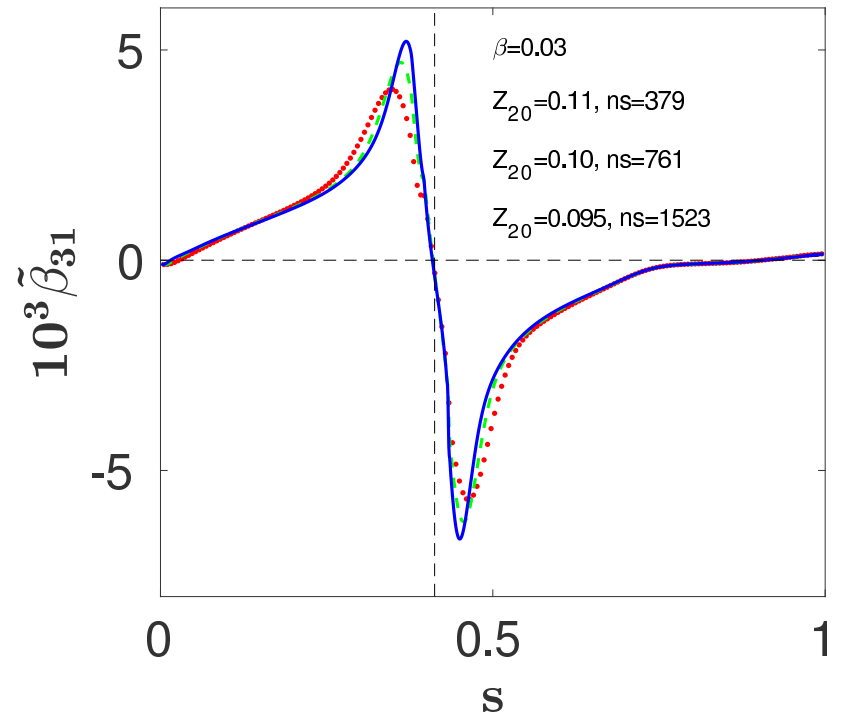

Figure 10. Various grid sizes and associated values of $Z_{m=2, n=0}$ render $\tilde{\beta}$ an odd function at the resonance. Please note the difference in vertical scale between Fig. 6 and here.

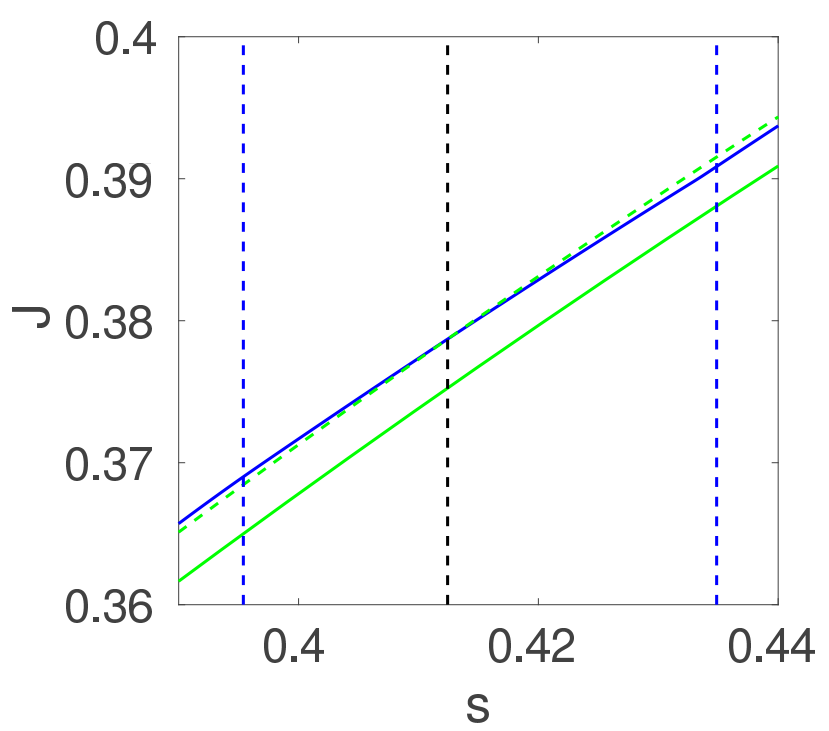

Figure 11. The toroidal current in case study b (upper solid line) and case study c (lower solid line) in the neighborhood of the resonance indicated by the middle vertical line; the left and the right vertical lines indicate the boundaries of the pressure profile flattening. The dashed line corresponds to the lower line shifted upwards to visualize the slightly lower derivative of the toroidal curret in case study b.

interesting region and finds them to be smooth.

Fig. 12 shows contours of $\tilde{\beta}$ for case study b). They are completely dominated by the resonant component $\tilde{\beta}_{31}$ before elimination of this current sheet and, after its elilmination, as has to be expected, mainly show the higher resonance $\tilde{\beta}_{62}$ with an amplitude of about 

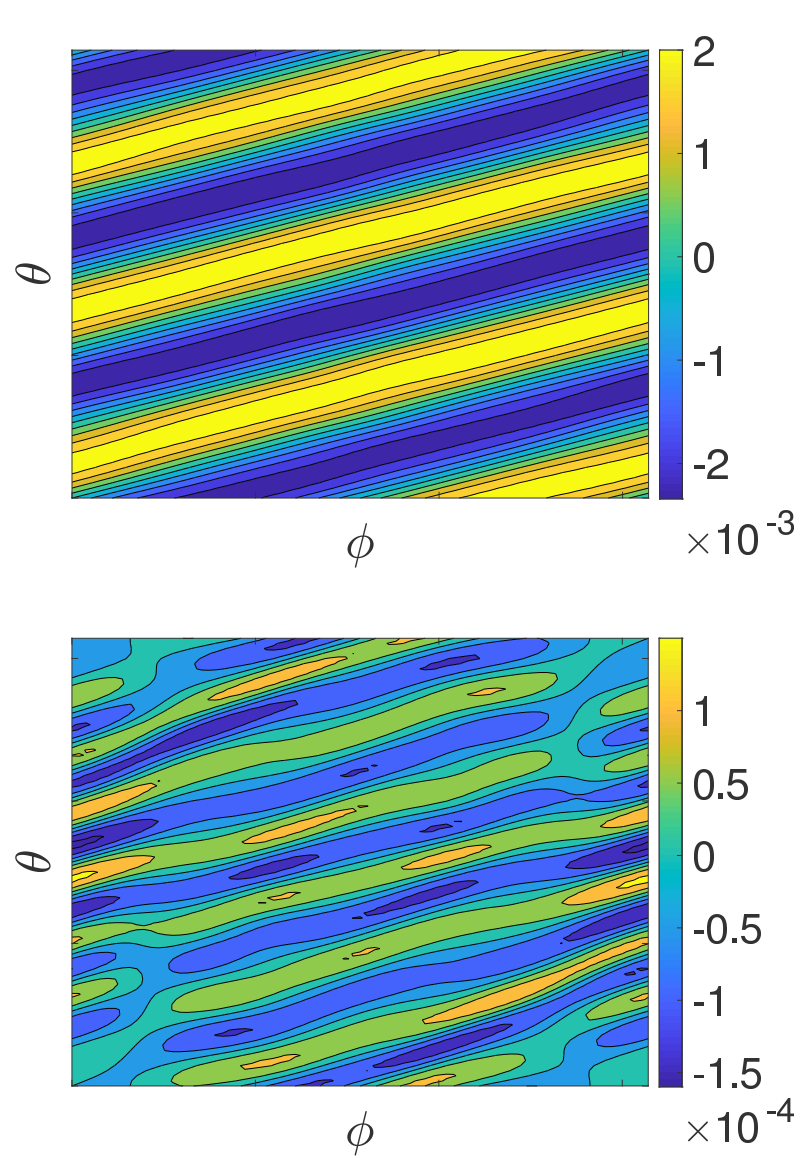

Figure 12. Contours of $\tilde{\beta}(\theta, \phi)$ at the resonance in case study $\mathrm{b}$ for $Z_{2,0}=0$, i.e. before eliminating the resonant current sheet $m=3, n=1$ (top), and after eliminating it (bottom). Please note the difference in the scales.

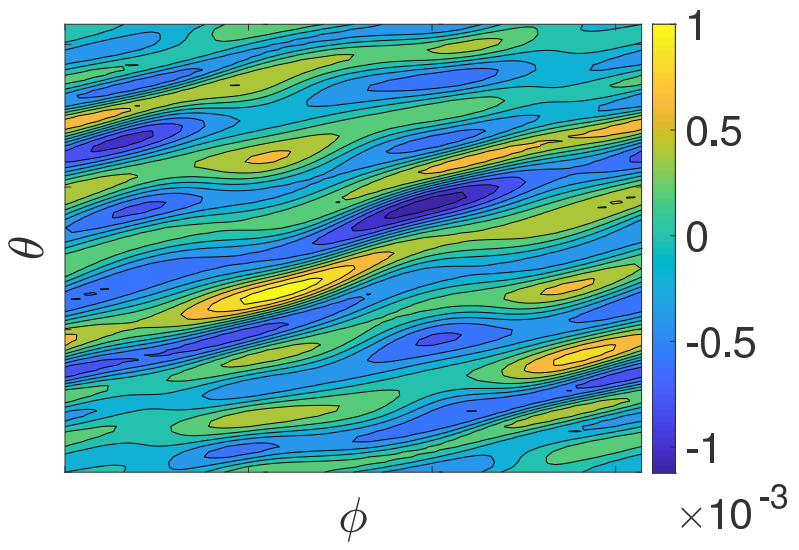

Figure 13. Contours of $\tilde{\beta}(\theta, \phi)$ at the resonance in case study c) for $Z_{2,0}=0.095$.

one order smaller than the original one of $\tilde{\beta}_{31}$.

Fig. 13 shows contours of $\tilde{\beta}$ for case study c) which again mainly show the higher resonance $\tilde{\beta}_{62}$ but with higher amplitude than in case study b)

\section{Discussion}

The results found above should be checked with $3 \mathrm{~d}$ equilibrium codes which do not assume nested magnetic surfaces as, by way of example, current versions of PIES [14] and HINT [15] as well as SIESTA [16] and SPEC [17].

The results obtained here suggest that current sheets in $3 \mathrm{~d}$ ideal-mhd equilibria can be avoided by adjusting the confinement geometry.

In case of a verification of these results further applications ot the procedure presented here can be investigated, by way of example, up to which order in poloidal and toroidal periodicity of a current sheet this procedure can be pursued. By way of example, an interesting case would be a high- $\beta$ equilibrium in W7-X in which $\iota=5 / 6$ occurs in the confinement region [18].

A successful design of a threedimensional toroidal confinement configuration will aim at a sufficiently well-behaved topological structure of its magnetic field at finite $\beta$. On the other hand, the perturbation of the property of quasi-axisymmetry of the configurations in case studies b) and c) will result in a study on the compatibility of quasi-axisymmetry and absence of current sheets.

\section{Acknowledgments}

This work has been carried out within the framework of the EUROfusion Consortium and has received funding from the Euratom research and training programme 2014-2018 under grant agreement No 633053. The views and opinions expressed herein do not necessarily reflect those of the European Commission. The support of Profs. S. Günter and P. Helander is gratefully acknowledged. Also, as for several decades, it is a great pleasure to acknowledge the use of Steven Hirshman's VMEC code.

\section{References}

[1] Hirshman S, van RIJ W and Merkel P 1986 Comp. Phys. Comm. 43143

[2] Reiman A et al 2015 Nucl. Fusion 55063026

[3] Loizu J et al 2015 Phys. Plasmas 22022501

[4] Loizu J et al 2015 Phys. Plasmas 22090704

[5] Loizu J et al 2016 Phys. Plasmas 23055703

[6] Lazerson SA et al 2016 Phys. Plasmas 23012507

[7] Boozer A 1980 Phys. Fluids 23904

[8] Nührenberg J and Zille R 1987 Theory of Fusion Plasmas 3

[9] Cary JR and Kotschenreuther M 1985 Phys. Fluids 281392

[10] Mikhailov M et al 2017 Nucl. Fusion 57064003

[11] Rummel Th et al 2012 IEEE on Applied Superconductivity 224201704

[12] Nührenberg J et al 1994 Theory of Fusion Plasmas 3

[13] Mercier C 1962 Nucl. Fusion 1962 Suppl.2 801

[14] Reiman A et al 1986 Comp. Phys. Com. 43157

[15] Hayashi T et al 1994 Phys. Plasmas 13262 
[16] Hirshman S et al 2011 Phys. Plasmas 1806204

[17] Hudson S et al 2012 Phys. Plasmas 19112502

[18] Mikhailov M et al 2011 Plasma Physics Reports 37391 